A History of Collective Living 



\section{Susanne Schmid}

\section{A History of Collective Living}

Forms of Shared Housing

Edited by

Susanne Schmid

Dietmar Eberle

Margrit Hugentobler

Edition Wohnen

ETH Wohnforum

ETH CASE

Birkhäuser

Basel 
